\title{
A method of operational effectiveness analysis for aerocraft based on L1 regularized logistic model
}

\author{
Tao Lin ${ }^{1,}$, Ke Zhang ${ }^{2, ~ b}$, Nai gang Cui ${ }^{3, ~ c}$, Zhen biao Tu, d \\ ${ }^{1}$ New technology building 401, Harbin Institute of Technology, Harbin, Heilongjiang, China \\ ${ }^{2}$ Fengtai District Yungang North, No. 40 hospital, Beijing, China \\ ${ }^{3}$ Main building 205, Harbin Institute of Technology, Harbin, Heilongjiang, China \\ ${ }^{4}$ Fengtai District Yungang North, No. 40 hospital, Beijing, China \\ altaodh@sohu.com, bzhangkehit6@yahoo.com, ccui_nai_gang@163.com, ${ }^{d}$ tuzhenbiao \\ @vip.sina.com
}

Keywords: operational effectiveness, L1 norm regularized, logistic model, aerocraft.

Abstract. A method of operational effectiveness analysis for aerocraft is proposed based on L1 regularized logistic model, for the problem of the operational effectiveness index is not easy to quantify analysis .The operational effectiveness of aerocraft is affected by many factors which relationship are complex, and index is difficult to quantitative analysis, when the aerocraft is in a complex battlefield environment. A set of analysis method and the analysis process for the study of the aerocraft operational effectiveness is provided. It uses machine learning methods to solve the problem of multi factors and complex relationship of operational effectiveness, by converted operational effectiveness sensitivity analysis to feature effectiveness judgment based on category. This method provides an effective method of tactical application for aerocraft penetration to seek the main influence factors from the whole.

\section{Introduction}

Battlefield environment has become increasingly complex, the aircraft penetration countermeasure process has become more intense, with rapid development of the defensive and offensive techniques as well as large-scale application of precision-guided weapons[3]. On one hand, the complex antagonistic environment is constituted by various interference factors and meteorological factors such as wind, rain, waves, ground clutter, sea clutter and so on, as well as the human factors which include a variety of intercepting weapons and electronic jamming equipment. On the other hand, with the improvement of the informationalized level and the diversification of operational pattern for aerocraft, The perception ability of the missile to the environment is significantly improved. At the same time, degree of dependence on battlefield information has also been strengthened, which make aerocraft to face a variety of threats through the whole process and all time of the combat. Thus it can be seen, the factors that affect the operational effectiveness of aerocraft are no longer simply attributed to the performance of aerocraft itself, but it needs to analyze the mutual influence relationships and interaction between aerocraft and battlefield environment through the whole process of combat, as for the increasingly informationalized level of aerocraft.

In order to explore the operational effectiveness of aerocraft which is in the complex battlefield environment, we need to make use of sensitivity analysis method to find the main factors which affect the operational effectiveness of aerocraft. At present, according to the scope of the sensitivity analysis, the common methods are divided into local and global sensitivity analysis. Local sensitivity analysis only examines the degree of the influence of a single attribute changes to the model. The main methods include sensitive coefficient, differential ratio method, automatic differential method, perturbation method etc. The global sensitivity analysis examines the overall impact of multiple attributes common changes to the model, then, it analyzes the effect of interaction between the attributes to the model output. The main methods include screening method, regression analysis, Fourier amplitude sensitivity test, response surface methodology, mutual information index method, Sobol index method and so on. 
In a complex confront environment, penetration and confrontation process of aerocraft has great uncertainty with game conditions during full time. Factors which may affect the effectiveness of penetration and confrontation are too many, and they have complex relationship. Obviously the methods of local sensitivity analysis cannot meet the needs. While, for analysis demands of operational effectiveness index sensitivity in different battle conditions, those methods of global sensitivity analysis which we have (Screening methods, regression analysis, etc.) also couldn't satisfy all the requirements.

\section{Sensitivity Analysis of Aerocraft Operational Effectiveness Based on machine learning}

As a computer technology, machine learning increasingly become one of the important fields of the practical, research and exploitation in computer science, in recent years. And it is widely used in sports, financial, Internet and other fields. By building a reasonable classification model, we can use the powerful computing to dig out the required information from the complex mass data.

There are more factors affecting the operational effectiveness of aerocraft, with the improvement of the level of information and intelligence of aerocraft. The traditional analytical methods have been difficult to describe the complex process of aerocraft. A typical test cannot fully reflect the different operational modes and the game process and results. Therefore, we need to consider a variety of combat aerocraft interaction style, environmental information and battlefield confrontation process.

For the problem of operational effectiveness analysis for aerocraft which is in the complex battlefield environment, combined with the advantages of machine learning, a method of aerocraft operational effectiveness analysis based on L1 norm regularized logistic model is proposed. It can solve the problem of penetration against effectiveness analysis for information aerocraft. The basically process of this method is as follows: First, we structure the logistic regression model based on the L1 regularized[1]; then, according to the aircraft combat process simulation test data in accordance with the logistic regression model input requirements, we collate and normalized process operational effectiveness index parameter to form data set, and the data set is divided into training set and cross checking set. Finally, according to the method of machine learning, the overall sensitivity is trained, and the main factors that affect the operational effectiveness and their weight are obtained from the overall situation. 


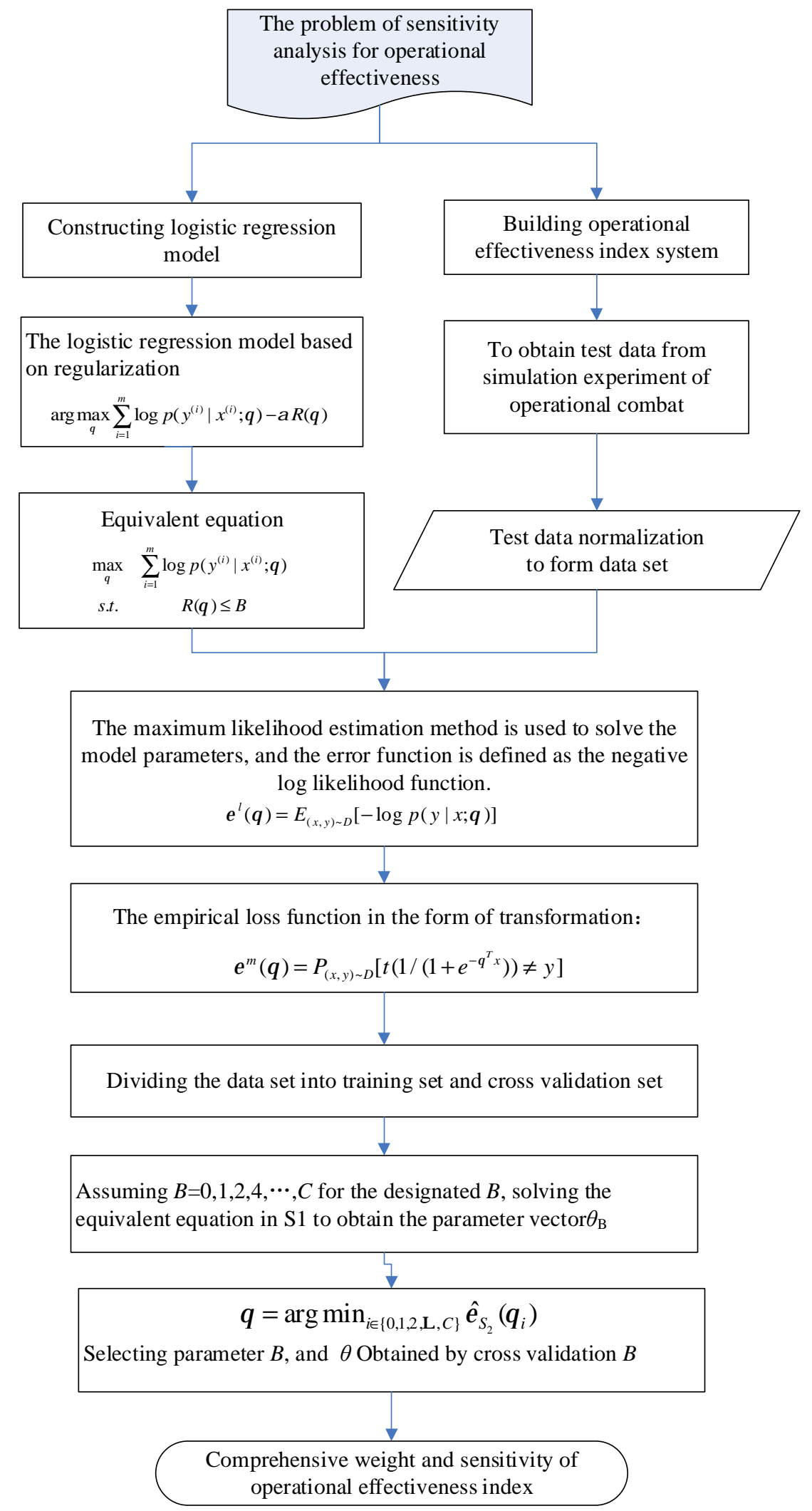

Fig.1 The sensitivity analysis process of aerocraft operational effectiveness based on machine learning

Logistic regression models: We use the following supervised learning model: we give a set of $m$ training samples to form a sample set $S . S=\left\{\left(x^{(i)}, y^{(i)}\right)\right\}_{i=1}^{m}$. All the samples obey the same distribution D. $x^{(i)} \in[-1,1]^{n}$ is the $n$ dimension input. $y^{(i)} \in\{0,1\}$ is the category label. In order to unify offset term, the last dimensional $x_{n}^{(i)}$ of vector $x^{(i)}$ is always set as 1 . In this case, logistic regression model [2] equation expressed as follows: 


$$
\begin{aligned}
& p(y=1 \mid x ; \theta)=\frac{1}{1+\exp \left(-\theta^{T} x\right)} . \\
& p(y=0 \mid x ; \theta)=\frac{1}{1+\exp \left(-\theta^{T} x\right)} .
\end{aligned}
$$

In Eq. 1 and Eq. 2, $\theta \in R^{n}$ is the weight vector. $\theta_{j}(j=1,2, \cdots, n)$ reflects the sensitivity of the $\mathrm{j}$-dimension of input vector to the output of the model.

Logistic model can be solved using the maximum likelihood estimation method[1], described in detail as follows:

Assume $p(y=1 \mid x)=\pi(x), p(y=0 \mid x)=1-\pi(x)$,

The likelihood function is

$$
\begin{aligned}
L(\theta) & =\sum_{i=1}^{m}\left[y^{(i)} \log \pi\left(x^{(i)}\right)+\left(1-y^{(i)}\right) \log \left(1-\pi\left(x^{(i)}\right)\right)\right] \\
& =\sum_{i=1}^{m} y^{(i)} \log \frac{\pi\left(x^{(i)}\right)}{1-\pi\left(x^{(i)}\right)}+\log \left(1-\pi\left(x^{(i)}\right)\right) \\
& =\sum_{i=1}^{m} y^{(i)} \theta^{T} x^{(i)}-\log \left(1-\exp \left(\theta^{T} x^{(i)}\right)\right)
\end{aligned}
$$

For the optimization objective function $L(\theta)$, using gradient descent algorithm or quasi-Newton method, evaluate the maximum value of $L(\theta)$, we can get the estimated value of $\theta$.

Operational effectiveness index system and operational simulation test data: According to the requirements of operational effectiveness index sensitivity analysis for aerocraft, index system of aerocraft operational effectiveness is built, and operational simulations have been carried out. We also completed sorting and normalization process of test data, finally, data set $S$ was formed .

Logistic regression models based on $\mathrm{L1}$ regularization: We suppose that we have a group aircraft attack and defense simulation test data, which means $m$ training samples, $S=\left\{\left(x^{(i)}, y^{(i)}\right)\right\}_{i=1}^{m}$. And the samples obey the same distribution. $x^{(i)} \in[-1,1]^{n}$ is corresponding factors which affect operational effectiveness index values, that is to say, there are $n$ factors used as the input parameters. $y^{(i)} \in\{0,1\}$ is the Normalized Operational Effectiveness, which can represent on damage probability of the target, and it is also category label. In order to unify offset term, set $x_{n}^{(i)}=1$. In this case, Logistic regression model equation is presented by Eq. 1 and Eq. 2.

In order to find the most important factor affecting operational effectiveness index more quickly, the regularization term was added. For logistic regression model added regularization term, we provides a unified description of the problem to optimize the formula for the parameter $\theta$.

$$
\underset{\theta}{\arg \max } \sum_{i=1}^{m} \log p\left(y^{(i)} \mid x^{(i)} ; \theta\right)-\alpha R(\theta)
$$

In Eq. $4, R(\theta)$ is a regular item, to punish excessive weight of the item. When $R(\theta) \equiv 0$, the above formula degenerates into standard logistic regression model. Select $R(\theta) \equiv\|\theta\|_{1}=\sum_{i=1}^{n}\left|\theta_{i}\right|$, so the above formula is L1 logistic regression model.

In Eq. $4, \alpha \geq 0$ is a mediation parameters, which is used to optimize the relationship between function fitting effect and the regular parameter punishment. In order to facilitate the analysis, the optimization problem of Eq. 4 is converted into the following: 


$$
\begin{array}{lr}
\max _{\theta} \sum_{i=1}^{m} \log p\left(y^{(i)} \mid x^{(i)} ; \theta\right) \\
\text { s.t. } \quad R(\theta) \leq B .
\end{array}
$$

After $\alpha$ is given, for each solution of the equation (4), there always exist $\beta$ to make the optimization problem of the solution of equation (5) be $\theta$. For the optimization problem of Eq. 5, using the maximum likelihood estimation method to solve the model parameters, and the error function is defined as the negative logarithm likelihood function[1, 2]。

$$
\varepsilon^{l}(\theta)=E_{(x, y) \sim D}[-\log p(y \mid x ; \theta)] \text {. }
$$

In Eq. 6, $(x, y) \sim D$ denotes test sample $(x, y)$ obey the distribution $\mathrm{D}$. When the data set $S$ is given, the loss function for a logarithmic form of experience is defined:

$$
\hat{\varepsilon}^{l}(\theta)=\hat{\varepsilon}_{s}^{l}(\theta)=\frac{1}{m} \sum_{i=1}^{m}-\log p\left(y^{(i)} \mid x^{(i)} ; \theta\right)
$$

So for the problem of 0/1 classification fault, Eq. 7 becames Eq. 8 .

$$
\varepsilon^{m}(\theta)=P_{(x, y) \sim D}\left[t\left(1 /\left(1+e^{-\theta T x}\right)\right) \neq y\right]
$$

In Eq. 8, $\mathrm{t}$ is the threshold function (When $z \geq 0.5, t(z)=1$. Otherwise, $t(z)=0$ )。

When the sample $\mathrm{m}$ is fixed, for any $\varepsilon>0, \delta>0, C>0,0<\gamma<1, K>1$, supposing there are $r$ indexs $1 \leq i_{1}, i_{2}, \cdots, i_{r} \leq n$, as well as the parameter vector $\theta^{*} \in R^{n}$, and the corresponding weight of $r$ indexs is not $0,\left|\theta_{i_{j}}\right| \leq K(j=1, \cdots, r)$, and $C>r K$. When $\hat{\theta}$ is close enough to $\theta^{*}$, the correct probability of the model will not below $1-\delta$ 。Now, $\varepsilon^{l}(\hat{\theta}) \leq \varepsilon^{l}\left(\theta^{*}\right)+\ni$, then $m=\Omega((\log n) \cdot \operatorname{poly}(r, K, \log (1 / \delta), 1 / \ni, C))$ 。 Under the precondition of L1 regularization, model accuracy and the required number of samples is logarithmic relationship.

Solving method of logistic regression model based on L1 regularization: The steps to analysis the $\mathrm{t}$ operational effectiveness indexes sensitivity for aerocraf using the L1 regularization logistic regression model $[7,8,9]$ are as follows:

1) The penetration simulation test data are sorted according to the input requirements of the logistic regression model, and the penetration index parameters are handled by normalized processing forming the data set $S$. The data set $S$ is divided into training set $S 1$ (including the above $(1-\gamma) m$ samples)and cross validation set $\mathrm{S} 2$ (including the rest of $\gamma m$ samples).

2 ) Assuming $B=0,1,2, \ldots, C$. For the specified $B$, we solve the equation (4) on $S_{1}$ to obtain $\theta_{B}$ 。

3 ) From all of the $\theta_{B}$ obtained in step 2, we select the one which makes $S_{2}$ be the smallest, i.e. $\theta=\arg \min _{i \in\{0,1,2, \ldots, C\}} \varepsilon_{S_{2}}\left(\theta_{i}\right)$ 。

By the cross validation to select the parameter $B$, the obtained vector $\theta$ is operational effectiveness index, i.e. the sensitivity of the vector $x$.

\section{Simulation test}

The influence factors of operational effectiveness for aerocraft under typical combat conditions: Under the condition of confrontation, a certain type of operational effectiveness as an example is taken to analysis the main factors influencing the aerocraft hit probability. According to the weights to sort the main factors, we assume that the aerocraft carrying radar seeker has the ability of 
maneuvering penetration and active electronic countermeasure. The aerocraft encounters the interception of anti-aerocraft and the interference of devise in the operational process. According to the set of battlefield environment and the fight scene, it shows the influence factors on the performance of the hit probability include the spacecraft themselves, natural environment factor, the threat to the environment factors and penetration strategy. And each aspect contains a number of factors in the process of actual combat. In order to simplify the simulation test, the factors that are directly related to the operational are chosen. Then, the attack-defense confrontation simulation system is constructed by using behavior modeling method. Finally, the feasible of operational effectiveness sensitivity analysis method based on L1 regularization is checked according to the simulation results.

Table 1 The influencing factors of aerocraft hit probability

\begin{tabular}{|c|c|c|c|}
\hline No. & Classes of Factors & Factors & Remarks \\
\hline 1 & \multirow{3}{*}{$\begin{array}{l}\text { The properties of } \\
\text { spacecraft }\end{array}$} & $\mathrm{Rm}$ & Firing range $[\mathrm{km}]$ \\
\hline 2 & & $\mathrm{Hm}$ & Flight height[m] \\
\hline 5 & & $\mathrm{Vm}$ & Flight speed $[\mathrm{m} / \mathrm{s}]$ \\
\hline 6 & \multirow{3}{*}{ Natural environment } & Weather & $\begin{array}{l}\text { The weather ( three conditions: sunny, rain, } \\
\text { fog) }\end{array}$ \\
\hline 7 & & Vwave & The wind (wind speed $[\mathrm{m} / \mathrm{s}]$ ) \\
\hline 8 & & IsClutter & Clutter (clutter or no clutter) \\
\hline 9 & \multirow{8}{*}{$\begin{array}{l}\text { Threats of the } \\
\text { environment }\end{array}$} & $\mathrm{X}$ & Air defense missile deployment location $\mathrm{x}$ \\
\hline 10 & & $\mathrm{Z}$ & Air defense missile deployment location $\mathrm{z}$ \\
\hline 11 & & T_Launch & Air defense missile launch time \\
\hline 12 & & $\mathrm{Td}$ & $\begin{array}{l}\text { Air defense missile interceptor strategy (one } \\
\text { intercept one or two intercept one) }\end{array}$ \\
\hline 13 & & $\mathrm{~N}$ & Air defense missile maximum overload \\
\hline 14 & & AngErr & $\begin{array}{l}\text { Air defense missile seeker angle measuring } \\
\text { error[rad] }\end{array}$ \\
\hline 15 & & DisErr & Air defense missile seeker ranging error $[\mathrm{m}]$ \\
\hline 16 & & $P_{j}$ & $\begin{array}{l}\text { The Interference power of shipboard } \\
\text { device }[w]\end{array}$ \\
\hline 17 & \multirow{4}{*}{ The penetration strategy } & $\mathrm{Cy}$ & Aerocraft maneuver cycle[s] \\
\hline 18 & & Range & The amplitude of aerocraft maneuvering $[\mathrm{m}]$ \\
\hline 19 & & $\mathrm{~T}_{\mathrm{mj}}$ & Aerocraft maneuver timing $[\mathrm{s}]$ \\
\hline 20 & & $P_{m j}$ & The active jamming power of aerocraft[w] \\
\hline
\end{tabular}

Simulation experiment and result analysis: Taking the factors in Table 1 as experimental factors, the experiment results are given by means of orthogonal test design method. In table 20, the hit probability of the aircraft is used as the output, and the vector $(x, y)$ is formed as the input of the 1 parameters. In the vector, $\mathrm{x}=(\mathrm{Rm}, \mathrm{Hm}, \mathrm{Vm}$, Weather, Vwave, IsClutter, X, Z, T_Launch,

$\mathrm{Td}, \mathrm{N}$, AngErr, DisErr, $\mathrm{P}_{\mathrm{j}}, \mathrm{Cy}$, Range, $\left.\mathrm{T}_{\mathrm{mj}}, \mathrm{P}_{\mathrm{mj}}\right)$

, $\mathrm{y}=\mathrm{P}_{\text {hit }}$, then the vector set $S=\left\{\left(x^{(i)}, y^{(i)}\right)\right\}_{i=1}^{n}$ is formed via mapping the $n$ sets data of the simulation experiment that have been normalized to the vector $(x, y)$ respectively. Considering the vector set as input, the main factors that 
affect the hit probability and their weights are obtained by machine learning. The simulation results are shown in figure 2.

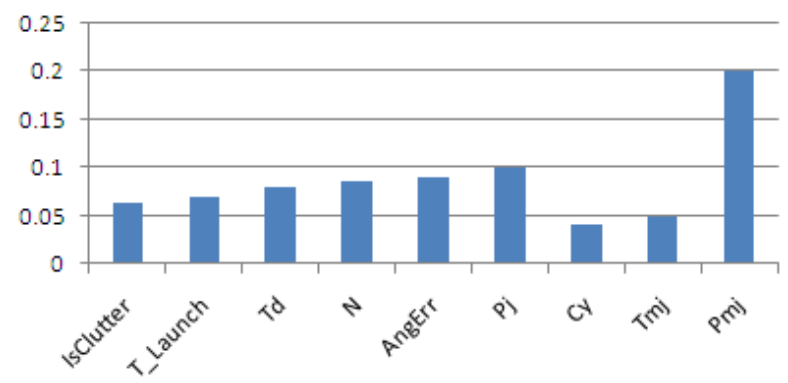

Fig.2 The main factors influencing aerocraft operational effectiveness

Figure 2 shows that the main factors influencing the aerocraft hit probability include clutter, air defense missile launch time, Air defense missile interceptor strategy, Air defense missile maximum overload, Air defense missile seeker angle measuring error, Aerocraft maneuver cycle, The Interference power of shipboard device, aerocraft maneuver timing, and The active jamming power of aerocraft. The nine main factors work together to determine the aerocraft hit effect directly, and the weights of other non-essential factors degrade to 0 .

From the results of semi-physical simulation and field test of aerocraft, the result obtained based on L1 regularization logistic model is more truly reflect the sensitive factors influencing the aerocraft operational effectiveness. The mathod provides a new method of operational effectiveness analysis for aerocraft in complex combat environment .

\section{Conclusion}

Considering complex aerocraft operational effectiveness against the environment as the research object, the paper studies the main factors that affect aerocraft operational effectiveness. Via building a reasonable regression model and choosing the appropriate regularization, the main factors influencing the combat effectiveness can be founded in the massive digital simulation test data using machine learning method. Typical test results show that the sensitivity analysis method of aerocraft operational effectiveness based on L1 regularization logistic regression model is feasible and effective. The paper provides a new method and approach for optimizing design of aerocraft and combat using research. And the method is important to promote the development of weapon equipments.

\section{Reference}

[1] Peter Harrington, Li Rui, Li Peng . Machine Learning in Action [M]. Beijing:People Post Press, 2013:73-84.

[2] Liu Zhenqiu, Jiang Feng, Tian Guoliang, et al.Sparse Logistic Regression with Lp Penalty for Biomarker Identification[J]. Statistical Applications in Genetics and Molecular Biology, 2007, 6(1):2-12.

[3] Zhang Ke, Liu Yong-cai, Guan Shi-yi. An investigation into the problem of evaluating combat effectiveness for missile weapon systems[J].Journal of Asronautics, 2002, 23 (2) , 58-59.

[4] Bai Hongtao, He Lili, Ouyang Dantong, et al.K-means on Commodity GPUs with CUDA[C]//Proc.of WRI World Congress on Computer Science and Information Engineering, [S.1.]:ACM Press, 2009:651-655.

[5] Mario Z, Michael G.Accelerating K-means on the Graphics Processor via CUDA[C]//Proc.of the 1st International Conference on Intensive Applications and Services.[S.1.]:IEEE Press, 2009:7-15. 
[6 Wang Si-fu, Liu Yong-cai, Guan Shi-yi, Zhang Ke. Study of Cooperation Effectiveness for UAV with Escort Free-Flight Decoys[J]. Journal of Asronautics, 2002, 23 (2) , 58-59.

[7] $\mathrm{Ng}$ A.Feature Selection, L1 vs L2 Regularization, and Rotational Invariance[C]//Proc.of International Conference on Machine Learning.New York, USA:ACM Press, 2004.

[8] Koh K, Kim S, Boyd S.An Interior-point Method for Large-scale L1-regularized Logistic Regression[J].Journal of Machine Learning Research, 2007, 1(8):1519-1555.

[9] Koh K, Kim S, Boyd S. An Interior-Point Method for Large-Scale L1 -Regularized Logistic Regression. Journal of Machine Learning Research, 2007, 8:1519-1555.

[10] Wu Yanrui, Wu Youli, Diao Xinghua, wood buildings, DingWei Battle Simulation Based Missile Weapon System Operational Effectiveness Evaluation [J]. Fire Control and Command Control, 2014.9. Vol. 39, No. 9, $19 \sim 22$. 